\title{
Grandes illusions et récits de vie
}

In: Politix. Vol. 7, N²7. Troisième trimestre 1994. pp. 183-188.

Citer ce document / Cite this document :

Damamme Dominique. Grandes illusions et récits de vie. In: Politix. Vol. 7, №27. Troisième trimestre 1994. pp. 183-188.

doi : 10.3406/polix.1994.1869

http://www.persee.fr/web/revues/home/prescript/article/polix_0295-2319_1994_num_7_27_1869 


\title{
Grandes illusions et récits de vie
}

\author{
Dominique Damamme
}

Université de Rouen

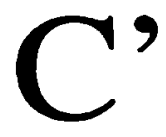

EST UN DES APPORTS des récits biographiques que de contribuer à la connaissance de la formation et de l'actualisation des dispositions, attitudes et représentations de soi des êtres sociaux. Mais parce que le moi est historique et relationnel, que les individus ne sont jamais transparents à eux-mêmes ${ }^{1}$, parce qu'en outre l'entreprise biographique, quand elle ne vise pas explicitement à la célébration de la personne, fait fond sur la représentation classique de l'individu ${ }^{2}$, entretenant ainsi l'illusion d'un sujet $^{3}$, ces dispositifs littéraires imposent, pour toutes ces raisons, qu'on questionne l'identité qu'ils accréditent et le sens de la vie qu'ils prétendent découvrir. Ce n'est qu'à la condition d'interroger la consistance de l'univers mental de l'individu et parallèlement d'appréhender le rapport du biographe à son objet qu'on pourra accorder quelque créance au portrait biographique et par conséquent aux représentations du monde et de soi de l'acteur.

Dans ses formes les plus routinisées, c'est-à-dire aussi dans sa forme la plus singularisante, l'entreprise biographique, à la suite d'une opération fondatrice de mise en énigme, se présente comme une tentative de dévoilement et de restitution du sens vrai d'une vie. Pourtant, le dispositif biographique, en histoire comme en sociologie, peut servir d'instrument à l'analyse d'un collectif, formation sociale, groupe social, communauté locale (L. Febvre, G. Duby, C. Ginzburg, N. Z. Davies, G. Levi, etc.). Si on ne peut donc attribuer au biographique des propriétés consubstantielles de décontextualisation ou une logique d'individualisation, reste que les biographies qu'on qualifiera de relationnelles par opposition aux biographies monadiques, pour marquer l'attention qu'elles portent aux interdépendances, sont de loin les plus rares ${ }^{4}$. C'est que l'entreprise historienne accorde privilège aux hommes egrands", aux -vies- d'artiste, de savant ou d'homme politique ; c'est aussi que la grandeur d'un individu implique l'individualité, et cela en un double sens : en appelant la singularisation, elle tend à l'autonomisation de l'acteur par rapport aux champs sociaux où son action s'inscrit ; en postulant l'existence d'un sujet, ou encore d'une "vie* tendue vers un accomplissement. Dans ces conditions, la tentative, a priori légitime, de constituer en un genre spécifique une hagiographie politique 5 risque, outre les difficultés de critérialisation, de focaliser la recherche sur les visées les plus visibles d'édification morale ou politique et de voiler par là même les procédés, conscients et inconscients,

1. Starobinski (J.), Jean-Jacques Rousseau, la transparence et l'obstacle, Paris, Gallimard, 1971.

2. Auerbach (E.), Mimesis, la représentation de la réalité dans la littéralure occidentale, Paris, Gallimard, 1968.

3. Bourdieu (P.), -L'illusion biographique., Actes de la recherche en sciences sociales, 62-63, 1986. 4. Elias (N.), La société de cour, Paris, Flammarion, 1985 ; de Certeau (M.), Histoire et psychanalyse entre science et fiction, Paris, Gallimard, 1987.

5. Certeau (M.) de, L'écriture de l'bistoire, Paris, Gallimard, 1975. 
d'individuation comme les effets d'exemplarité qui découlent de la position centrale faite à un sujet mis en place du roi.

L'exception d'une edestinée- (et la grandeur qui la consacre) ne va cependant pas de soi ; elle demande à être expliquée tant elle est énigmatique, à la limite incompréhensible : comment en effet rendre compte rationnellement de qualités extraordinaires ou même de dons hors de l'ordinaire. Dans la culture populaire ${ }^{1}$, comme pour le sens commun savant, les actes comptent ou pèsent moins que la personne : ils n'ont de réalité et de signification que rétrospectivement, rapportés à leur terme, à leur cause finale, la personne, ou comme effectuations d'une essence. La compréhension est donc donnée par une mise en sens par le biais d'un stéréotype. N. Heinich a montré à propos de Van Gogh ${ }^{2}$ comment les figures du héros, du saint, du génie ou du martyr avaient pu servir de support à des célébrations de nature opérationnaliste, attachées aux actes et aux ouvres, ou personnalistes comme dans le cas du saint sacrificiel, rappelant par là même comment ces topot contribuent à condenser le flux infini des paroles et des actions d'un individu en leur conférant une configuration stable et unitaire.

L'illusion biographique s'enracine bien souvent - mais il est aussi des exemples inverses, Van Gogh précisément ${ }^{3}$ - dans le travail de définition et de présentation de soi des êtres sociaux. Cette croyance, et les biais qui en découlent, est d'autant mieux fondée qu'elle s'adosse à des prétentions explicites. Si le dispositif biographique, dans son quotidien historique et journalistique, continue de véhiculer de manière aussi prégnante une idéologie du sujet, cette situation tient au fait qu'elle est proposée, pour ne pas dire imposée, par l'acteur lui-même. Le travail d'identification et de catégorisation effectué par le biographe ne fait fréquemment que refléter ces offres identitaires. Les catégories ou les schèmes explicatifs qu'il utilise sont en quelque sorte offerts par le biographé, sous forme d'identités stratégiques mais aussi de projections narcissiques de l'enfance, de traductions symboliques d'une ambition ou d'une carrière en gésine, mais aussi d'investissements de modèles sociaux et politiques valorisés - le chef, le héros, le grand homme. C'est, nous semble-t-il, en rapprochant la construction biographique des élaborations identitaires des acteurs, en voyant que le biographique ordinaire repose sur une sorte de contrat entre l'acteur, l'auteur et le lecteur - dans la mesure où ces récits répondent aux attentes culturelles du public - qui pactisent entre eux sur quelques topol fondamentaux, véritables dispositifs culturels, institutions sociales pour E. Durkheim, ssignifications institutionnalisées* de la culture pour J. Bruner, organisant la connaissance des choses et par là leur réalité et leur légitimité, que l'on peut comprendre la dureté et la résistance de ce type d'outillage à la fois mental et social.

S'intéresser aux identifications en lesquelles les individus se projettent et aux identités dont ils se dotent, suppose - comme le montre l'usage des stéréotypes - l'analyse des modèles portés par la culture, et particulièrement par la culture scolaire. On pense ici aux remarques de $\mathrm{K}$. Marx sur l'invention de Rome par les révolutionnaires de 1789 : "Même quand ils [les hommes] semblent occupés à se transformer, eux et les choses, à créer quelque chose de

1. Bruner (J.), Car la culture donne forme à l'esprit, Paris, Eshel, 1991.

2. Heinich (N.), La gloire de Van Gogh. Essai d'anthropologie de l'admiration, Paris, Minuit, 1991. 3. Van Gogh, Lettres à Théo, Paris, Gallimard, 1988. 
tout à fait nouveau, c'est précisément à ces époques de crise révolutionnaire qu'ils évoquent craintivement les esprits du passé, qu'ils leur empruntent leurs noms, leurs mots d'ordre, leurs costumes, pour apparaître sur la nouvelle scène de l'histoire sous ce déguisement respectable et avec ce langage emprunté 1 . Travail de résurrection que $H$. Rosenberg qualifiait de atradition du nouveau», retour au passé pour la création d'un présent dont Rome au XVIIle et au XIXe siècles a offert la matrice historique, à la fois dispositif politique (la République, l'égalité citoyenne), mais aussi creuset de modèles de grandeur politique et d'excellence sociale qui ont peuplé l'horizon d'attente des lecteurs de Montesquieu et de Rousseau². Que la figure de législateur ou d'instituteur d'un peuple, présente dans L'Esprit des lois et dans le Contrat social, s'impose pendant la Révolution ne relève nullement d'un hasard ${ }^{3}$. Avec plus de force et de visibilité qu'à l'ordinaire, c'est dans les moments de crise d'un système social ${ }^{4}$, lorsque les individus sont confrontés à de brusques changements politiques et sociaux, à des transformations de leur condition sociale et à des ruptures identitaires ${ }^{5}$, que se répandent ces formes culturelles alternatives aux formes dominantes, d'autres "mondes", offrant de nouveaux schèmes d'interprétation, de nouveaux modèles d'action mais aussi des figures nouvelles d'identification, en somme tout un ensemble de schèmes intellectuels et pratiques, tout un stock de modèles d'action et d'images de soi qui orientent significativement la conduite de ces prétendants en quête de renommée, tel Saint-Just, en position de les actualiser.

L'information biographique est encore susceptible d'éclairer le travail d'autoanalyse de l'acteur et les ressais* de définition et de stylisation de la personne, tous phénomènes qui ne sont pas propres à la vie politique mais qui sont impliqués par toute vie publique et toute prétention à une vie publique. Parmi de multiples exemples possibles de ce travail de soi sur soi, on évoquera la Théorie de l'ambition d'Hérault de Séchelles (écho au Livre du courtisan de B. Gracian) ou les pages du Fil de l'épée (1928) dans lesquelles Charles de Gaulle, à travers le portrait de l'homme de caractère, dresse une théorie intéressée du charisme, prédestination qui touche certains hommes, mais aussi en l'occurrence prétention à la possession de cette grâce : "Face à l'événement, c'est à soi-même que recourt l'homme de caractère. Son mouvement est d'imposer à l'action sa marque, de la prendre à son compte, d'en faire son affaire [...]. L'homme de caractère confère à l'action sa noblesse; sans lui morne tâche d'esclave, grâce à lui, jeu divin du héros [...]. Fait affectif, suggestion, impression produite, sorte de sympathie inspirée aux autres, le prestige dépend, d'abord, d'un don élémentaire, d'une aptitude naturelle qui échappent à l'analyse. Le fait est que certains hommes répandent, pour ainsi dire de naissance, un fluide d'autorité dont on ne peut discerner au juste en quoi il consiste et dont on s'étonne parfois tout en subissant les effets* ${ }^{6}$. Ces stratégies d'identité, qui sont aussi des *identités stratégiques ${ }^{7}$, ne sont pas

1. Marx (K.), Le dix-buit Brumaire de Louis-Napoléon, Paris, Editions sociales, 1968.

2. Brockliss (L.W.B.), French Higher Education in the 17 th and 18th centuries. A Cultural History, Oxford, Clarendon Press, 1987.

3. Vidal-Naquet (P.), - Tradition de la démocratie grecque., in Finley (M.), Démocratie antique et démocratie moderne, Paris, Payot, 1976.

4. Durkheim (E.), De la division du travail social, Paris, PUF, 1991.

5. Pollac187

(M.), -La gestion de l'indicible., Actes de la recherche en sciences sociales, 62-63, 1986.

6. De Gaulle (C.), Le fil de l'épée, Paris, 10/18, 1962 (1ère édition Berger-Levrault, 1932), p. 53-54 et 77

7. Collovald (A.), -Identité(s) stratégique(s)•, Actes de la recherche en sciences sociales, 73, 1988. 
sans effet et sont parfois même si grosses d'effets que l'acteur a du mal à s'en débarrasser. C'est que toute personne (publique) se trouve progressivement enfermée dans des objectivations, façade et face, dont elle est à la fois le producteur et le produit. En un sens, l'homme politique est un auteur en quête d'un personnage : il se sert de typifications comme celles de militant, de parlementaire, voire d'homme d'État, qui contribuent à rendre logiquement et chronologiquement cohérentes ses actions, à ses yeux et aux yeux des autres. À nouveau, le général de Gaulle se donne en exemple : Même le caractère quelque peu mythique dont on décore mon personnage contribue à répandre l'idée que des obstacles, par tous autres infranchissables, vont s'aplanir devant moi [...]. Me voici, toujours contraint par l'exceptionnel crédit que me fait le peuple français, me voici, obligé autant que jamais d'être ce de Gaulle à qui tout ce qui arrive au-dedans et au-dehors est personnellement imputé, dont chaque mot, et chaque geste, même quand on les lui prête à tort, deviennent partout des sujets de discussion dans tous les sens et qui, nulle part, ne peut disparaître qu'au milieu des clameurs ardentes. Éminente dignité du chef, lourde chaîne du serviteur." Ces productions publiques d'un soi que l'acteur contribue pour partie à façonner, et, pour une autre part, nées de l'amour de ses fidèles, une fois bien fondées, lui deviennent comme extérieures et pèsent sur son activité. Elles lui collent à la peau, tout comme le succès d'un comédien l'enferme dans une composition. Constructions dotées d'une réalité autonome, ces objectivations constituent des contraintes d'image et donc d'identité. Elles valent comme des devoirs, elles impliquent la fidélité à soimême, le point d'honneur, l'obligation de ne pas déchoir. L'homme politique est ainsi souvent condamné à la reproduction; il est sommé d'être à la hauteur de la définition qu'il s'est donnée et qu'il se donne parce que les autres la lui ont donnée, en tout cas, à ne pas trop s'écarter du personnage qui constitue sa personne. En d'autres termes, l'identité peut devenir, au "cinéma" comme sur la scène politique, une entrave à la carrière : par exemple, Pierre Mendès France ne peut pas, ni en 1968 ni en 1969, faire personnellement acte de candidature à la présidence d'une République dont il a dénoncé le caractère autoritaire (mais cela ne gêne aucunement $F$. Mitterrand) ; Pierre Mendès France ne peut pas, en tant qu'autorité morale, se livrer, comme d'autres, à des actes qui pourraient être interprétés comme des manœuvres "politiciennes" contraires à sa réputation (à son identité) d'intégrité (passer des alliances contre-nature* pour emporter la direction d'un parti comme le fait $F$. Mitterrand). Les objectivations emportent ainsi des règles normatives qui interdisent le recours aux règles pragmatiques du métier politique, condamnent un éventuel retour à la politique active et obligent à se satisfaire d'une carrière "morale».

Prendre en compte les représentations de soi, c'est, dans la mesure où l'identité ne constitue pas une formation stable mais plutôt une somme de "réalités", addition de moments dans la trajectoire de l'individu et de figures relevant de plusieurs univers sociaux ${ }^{2}, s^{\prime}$ attacher au travail de rapiéçage auquel l'individu se livre pour intégrer ces différentes faces en un ensemble

1. De Gaulle (C.), Mémoires d'Espoir, Paris, Le Livre de Poche, 1973, p. 39-40 ; * Je crois que si l'Histoire a un sens, elle n'en est pas moins, dans une certaine mesure, malléable... Si les hommes auxquels le pouvoir est confié interprètent convenablement la réalité historique à laquelle ils sont confrontés, ils peuvent favoriser des accouchements, les rendre moins pénibles, moins douloureux; ou au contraire freiner tel ou tel progrès. (Lacouture (J.), Mendès France, Paris, Seuil, 1981).

2. Strauss (A.), Miroirs et masques, Paris, Métailié, 1992. 
présentable, suffisamment crédible aux yeux des autres comme à ses propres yeux. Cette identité, soumise à des épreuves de réalité qui scandent la "carrière* de l'acteur ${ }^{1}$ - épreuves de vérité confirmatoires ou dénégatrices générant un état de félicité ou de désillusion -, est l'objet d'un raccommodage identitaire que vient conforter le mécanisme de la dissonance cognitive ${ }^{2}$. L'analyse des représentations de soi est alors confrontée à des dispositions equi tiennent" et parfois à un schème de la vocation particulièrement résistant. Certains individus affirment une certitude de soi, une assurance en leur avenir telle qu'ils réussissent à imposer l'image d'une vie pleinement maîtrisée, toute entière tendue vers la réalisation de leur être. De même, certains individus manifestent une croyance si ancrée dans leur mission, voire dans leur prédestination, que, se faisant les apôtres de leur destin, ils semblent s'en faire aussi les agents ${ }^{3}$. Les biographes de Pierre Mendès France attestent ainsi que son existence a été *affectée à son destin politique. Il ne le voyait pas comme une carrière mais comme une mission [...]. Il avait tout jeune rêvé d'une vie pleinement dévouée à une grande œuvre ${ }^{4}$. "Je crois en moi», fait dire en une sorte de credo, Vigny à Stello, figure et double du poète. .Il est évident que Dieu a son idée sur moi, car je suis un vrai miracle à mes yeux. Je ne puis pas comprendre, autrement que par un souffle de Dieu, l'inconcevable popularité dont je jouis ici», écrit Lamartine dans une lettre. Ailleurs, il déclare : “Croyez-moi bien, j'ai l'instinct des masses $^{5}$. Autre exemple, Charles de Gaulle, fabulant dans sa quinzième année sur son avenir militaire, s'imagine qu'en 1930, l'Europe, irritée du mauvais vouloir et des insolences du gouvernement, déclara la guerre à la France. Trois armées allemandes franchirent les Vosges [...]. Le général de Gaulle fut mis à la tête de 200000 hommes [...]. Que le biographe Lacouture lise là une anticipation prophétique du futur ${ }^{6}$ — $* \grave{A} 15$ ans, Charles se voit, se sait déjà le général de Gaulle» - importe à la fois beaucoup et peu. Le procédé importe parce que, instituant un brouillon d'adolescent en indice vocationnel, le biographe de Charles de Gaulle pose directement la question du statut à donner aux "grandes illusions"; il importe aussi parce qu'il montre comment un choix professionnel, au demeurant tout à fait conforme aux attentes d'un milieu social, peut être transmué en preuve d'une vocation et fonder l'idée d'une vie voulue et accomplie. Et en même temps, cette machinerie biographique qui transforme en archive ce qui n'était sans doute qu'un jeu ne change rien au problème, car s'agirait-il d'une pure construction, elle ne délivrerait pas la recherche de l'obligation d'étudier à côté de l'avenir objectif l'avenir souhaité, nécessité s'imposant quelles que soient les formes prises par ces projections et ces anticipations, et peut-être plus encore s'il s'agit de rêves de gloire devenus réalité ou vécus et appréhendés comme étant devenu la réalité. Pour reprendre l'exemple du de Gaulle de J. Lacouture, il y a bien là invention rétrospective de la preuve d'une vocation ou d'une grâce, mais cette

\footnotetext{
1. Becker (H. S.), Outsiders, Paris, Métailié, 1985. On peut penser aussi à L'Éducation sentimentale de Flaubert. Si cette ligne d'interprétation qui pense l'identité comme dotée d'une stabilité toute relative tend progressivement à s'imposer, sans doute est-ce là un effet du développement de la différenciation sociale, de la scolarisation et de la mobilité sociale (ascendante, descendante, transfert du pôle économique au pôle intellectuel).

2. Dont il faudrait questionner le fonctionnement plus sérieusement qu'on ne l'a fait jusqu'à présent.

3. Merton (R. K.), Eléments de théorie et de méthode sociologique, Paris, Plon, 1965.

4. Par exemple, Lacouture (J.), Mendès France, op. cit., p. 50.

5. Benichou (P.), Les mages romantiques, Paris, Gallimard, 1988, p. 41 et 47.

6. cf. dans ce numéro l'article de Gaïti (B; ${ }^{\circ}$, «Jean Lacouture biographe».
} 
déconstruction n'abolit pas l'épisode en cause en tant que signe d'une ambition : elle éclaire un procédé historiographique, elle ne clôture pas l'enquête. Précisément, c'est face à ces dispositions socio-psychiques qui résistent aux changements ${ }^{1}$ et produisent des attitudes et des représentations durcies que s'impose l'obligation de préciser les contours de la notion d'habitus $^{2}$ - théorie de histoire sociale incorporée par l'acteur - et son articulation avec l'analyse de s'épigenèse de l'identité ou du "roman familial. de l'individu ${ }^{3}$.

1. Même si elles aussi finissent par se modifier en fonction des systèmes d'interdépendance au elles s'inscrivent. Voir Lacroix (B.), L'utopie communautaire, Paris, PUF, 1981.

2. Bourdieu (P.), Réponses, Paris, Seuil, 1992, p. 103. Le concept d'habitus ne prétend pas former, au moins dans son acception originaire, la totalité des déterminants des représentations et de l'action. Il faut compter d'une part avec la structure psychique, d'autre part sa compétence., sa propension au calcul et son travail d'auto-analyse.

3. Erickson (E.), Adolescence et crise, la quête de l'identité, Paris, Flammarion, 1972, et Robert (M.), Roman des origines, origine du roman, Paris, Gallimard, 1972. 